\title{
TRANSIENT VIBRATIONS OF AN ELASTIC CYLINDER INSERTED IN THE ELASTIC MEDIUM
}

\author{
Heorgij SULYM*, Imre TIMAR*, Ihor TURCHYN"* \\ ${ }^{*}$ Faculty of Mechanical Engineering, Bialystok University of Technology, Wiejska Str., 45C, 15-351 Bialystok, Poland \\ ${ }^{* *}$ Faculty of Engineering, University of Pannonia, Egyetem str., 10, H-8200 Veszprem, Hungary \\ ***idstryhach IAPMM, NANU, Naukova str., 3b, 79060 Lviv, Ukraine \\ sulym@pb.edu.pl, timari@almos.vein.hu, ihorturchyn@gmail.com
}

received 4 May 2015, revised 11 May 2016, accepted 16 May 2016

\begin{abstract}
Using method of Laguerre polynomials we have obtained the solution of the dynamic problem of the theory of elasticity for elastic cylinder inserted into massive body modeled as a space. The source of non-stationary processes in composite is high intensity force load of the inner surface of the cylinder. On the surface separation of materials of space and cylinder the conditions of ideal mechanical contact are satisfied. The solution is obtained as series of Laguerre polynomials, which coefficients are found from recurrent relations. The results of numerical analysis of transient stress-strain state in elastic space with cylindrical insertion might be used for the technological process of hydraulic fracturing during shale gas extraction.
\end{abstract}

Key words: Transient Wave Propagation, Nongomogeneous Medium, Analytical Solution, Laguerre Polynomials

\section{INTRODUCTION}

The common tendency of the development of modern technology and engineering is the elaboration and wide usage of new structural materials. Some of the most perspective new materials are composite materials, that are characterized by sheeting and significant discrepancy between mechanical features of structural sheets.

One of the most common methods of research of mechanical fields in composite bodies and spaces is homogenization of their features with further research of their behaviour as hypothetically homogeneous structures. With such approach we can simplify the general problem definition and use well-known methods of research of mechanical fields in homogeneous bodies. Still, using this approach we very often cannot authentically define qualitative and quantitative features of the processes in the very composite that are caused by its nonhomogeneity (Theotokoglou and Stampouloglou, 2008; Zhang and Hasebe, 1999).

The other approach in which an internal nonhomogeneity and interaction between separate parts of the composite are taken into account causes a consideration of separate problems for each composite element with further regard for their contact conditions (de Monte, 2006; Yin and Yue, 2002). Within this approach it is possible to account the real stress-strain state in every layer and define some peculiar features of the transformation of mechanical fields on the section surfaces. In case of flat-layered or spherelayered body for solving the received problems the Laplace integral transform was successfully used (Liu and Qu, 1998, Sulym et al., 2013, Wang et al., 2002). However, for inhomogeneous cylindrical bodies the direct usage of this transformation creates great difficulties of the transition from transforms to originals (Lu, et al., 2006). Specially, it is about the cases when a cylinder is inserted in elastic space - because of the phenomenon of vibration damp- ing we need to find complex roots of the complicated transcendental equation. Therefore, a lot of authors use either numerical methods of inversion of the Laplace integral transform (Dai and Wang, 2005;) or direct numerical methods (Onyshko and Senyuk, 2009; Savruk et al., 2008; Sladek et al., 2008).

This research aims at the elaboration of analytical method of finding the solution to dynamic axisymmetric problems of the theory of elasticity for cylinder included in elastic space, the investigation of transient stress-strain state in elastic space with cylindrical insertion, caused by impact load of its boundary surface. Method of solution based on the use to the problem of Laguerre integral transformation (Sulym and Turchyn, 2012; Turchyn and Turchyn, 2013).

\section{PROBLEM FORMULATION}

Now we consider the dynamic problem of the theory of elasticity for inserted into elastic space of cylinder with excellent mechanical properties of the medium (Fig. 1).

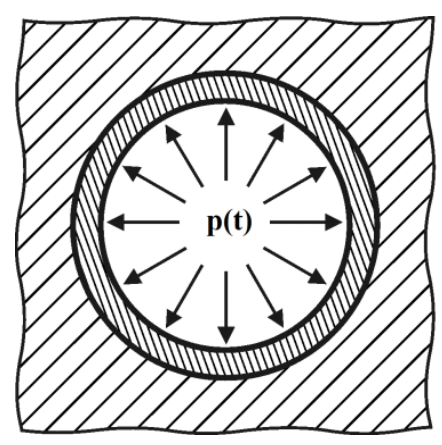

Fig. 1. Scheme of the problem 
The source of non-stationary processes in composite is high intensity force load of the inner surface of the cylinder.

In order to identify the stress and strain field in the composite, assuming that on the surfaces of cylinder and elastic medium conditions of ideal mechanical contact are true, we should find the solution to the initial-boundary problem:

$\rho^{-1} \partial_{\rho}\left(\rho \partial_{\rho} u^{(i)}\right)-\rho^{-2} u^{(i)}-\tilde{c}_{i}^{2} \partial_{\tau}^{2} u^{(i)}=0, \quad i=1,2$

$\sigma_{\rho \rho}^{(1)}=-p^{*}(\tau), \quad \rho=\rho_{0} ; \quad u^{(2)}=0, \rho \rightarrow \infty$;

$u^{(1)}=u^{(2)}, \sigma_{\rho \rho}^{(1)}=\sigma_{\rho \rho}^{(2)} \rho=1$;

$u^{(i)}=\partial_{\tau} u^{(i)}=0, \quad \tau=0, \quad i=1,2$,

where: $\rho=r / R_{1}-$ non-dimensional radial variable of the cylindrical coordinate system; $R_{0}, R_{1}$ - accordingly, radiuses of the inner and the external surface of the cylinder, $u^{(i)}(\rho, \tau)-$ displacment to the radial motion $(i=1$ - in cylinder, $i=2-$ in elastic medium; $\tilde{c}_{i}=\frac{c_{1}}{c_{i, 1}}, \quad \tau=\frac{c_{1} t}{R_{1}}-$ non-dimensional time; $c_{i, 1}$ - the longitudinal waves propagation velocities in the material of cylinder other elastic medium; $\sigma_{\rho \rho}^{(i)}(\rho, \tau)$ - radial stresses in the cylinder other elastic medium, that are determined by Hook's law:

$\sigma_{\rho \rho}^{(i)}=\mu_{i}\left[\kappa_{i}^{2} \partial_{\rho} u^{(i)}+\left(\kappa_{i}^{2}-2\right) \frac{u^{(i)}}{\rho}\right]$

where: $\kappa_{i}^{2}=\frac{\lambda_{i}+2 \mu_{i}}{\mu_{i}} ; \lambda_{i}, \mu_{i}-$ elastic constants.

\section{SOLUTION OF THE PROBLEM}

We will search for the problem (1)-(4) solution in the class of functions that belong to the space $L_{2}(0, \infty ; \lambda \exp (-\lambda \tau))$, i.e. for which the condition:

$\left\|u^{(i)}(\rho, \tau)\right\|^{2}=\lambda \int_{0}^{\infty} \exp (-\lambda \tau)\left|u^{(i)}(\rho, \tau)\right|^{2} d \tau<\infty$

is true, where $\lambda>0$ some number (scaled multiplier). Then, the functions $u^{(i)}(\rho, \tau)$ can be showed as a series of Laguerre polynomials:

$u^{(i)}(\rho, \tau)=\lambda \sum_{n=0}^{\infty} u_{n}^{(i)}(\rho) L_{n}(\lambda \tau)$,

where:

$u_{n}^{(i)}(\rho)=\int_{0}^{\infty} \exp (-\lambda \tau) u^{(i)}(\rho, \tau) L_{n}(\lambda \tau) d \tau$,

and $\mathrm{L}_{\mathrm{n}}(\lambda \tau)$ - Laguerre polynomials.

Further we will consider the formula (7) as integral transform of the function, and a series (6) - as the inversion formula of this transform.
Now we multiply the equation (1) on the conversion core $\exp (-\lambda \tau) L_{n}(\lambda \tau)$ and integrate the obtained expression according the variable $\tau$ in the interval $[0, \infty)$. Accounting the equation (7) and the initial conditions (4), after the integration by parts we will obtain:

$$
\begin{aligned}
& \rho^{-1} d_{\rho}\left(\rho d_{\rho} u_{n}^{(i)}\right)-\rho^{-2} u_{n}^{(i)}-\omega_{i}^{2} u_{n}^{(i)}= \\
& =\omega_{i}^{2} \sum_{m=0}^{n-1}(n-m+1) u_{m}^{(i)}, \quad i=1,2 ; \\
& \sigma_{\rho \rho, n}^{(1)}=-p_{n}^{*}, \rho=\rho_{0}, u_{n}^{(2)}=0, \rho \rightarrow \infty \\
& u_{n}^{(1)}=u_{n}^{(2)}, \sigma_{\rho \rho, n}^{(1)}=\sigma_{\rho \rho, n}^{(2)}, \rho=1,
\end{aligned}
$$

where $\omega_{i}=\lambda \tilde{c}_{i}$.

The solution to the triangular sequence of ordinary differential equations can be written as on algebraically convolution:

$u_{n}^{(i)}(\rho)=\sum_{j=0}^{n}\left[C_{n-j}^{(i)} G_{j}\left(\omega_{i} \rho\right)+D_{n-j}^{(i)} W_{j}\left(\omega_{i} \rho\right)\right]$.

Here are linearly independent fundamental solutions of the sequence (8), which we can represent as:

$$
\begin{aligned}
& G_{j}(x)=\sum_{p=0}^{j} a_{j, p} \frac{(x)^{p}}{2^{p} p !} \mathrm{I}_{p+1}(x) ; \\
& W_{j}(x)=\sum_{p=0}^{j} a_{j, p} \frac{(-x)^{p}}{2^{p} p !} \mathrm{K}_{p+1}(x),
\end{aligned}
$$

where: $I_{p}(x)$ and $K_{p}(x)$ - Bessel's modified functions, and coefficients $\mathrm{a}_{\mathrm{j}, \mathrm{p}}$ satisfy recurrence relations:

$a_{j, p+1}=\sum_{k=p}^{j-1}(j-k+1) a_{k, p}$,

$j=1,2, \ldots, p=\overline{0, j-1}$.

Accounting the conditions on infinity (2) and a view of fundamental solutions (12), we obtain that:

$C_{j}^{(2)} \equiv 0, j=0,1,2, \ldots$

The direct solutions stuffing (11) into conditions (9)-(10) leads to correlations, which after some transformations can be represented as recurrent sequences of systems of linear algebraic equations:

$\left(\begin{array}{lll}b_{1,1} & b_{1,2} & 0 \\ b_{2,1} & b_{2,2} & b_{2,3} \\ b_{3,1} & b_{3,2} & b_{3,3}\end{array}\right)\left(\begin{array}{l}C_{n}^{(1)} \\ D_{n}^{(1)} \\ D_{n}^{(2)}\end{array}\right)=\left(\begin{array}{l}H_{n, 1} \\ H_{n, 2} \\ H_{n, 3}\end{array}\right)$,

where:

$$
\begin{aligned}
b_{1,1} & =\kappa_{1}^{2} \omega_{1} I_{0}\left(\omega_{1} \rho_{0}\right)-\frac{2}{\rho_{0}} I_{1}\left(\omega_{1} \rho_{0}\right) ; b_{1,2}=-\kappa_{1}^{2} \omega_{1} K_{0}\left(\omega_{1} \rho_{0}\right)-\frac{2}{\rho_{0}} K_{1}\left(\omega_{1} \rho_{0}\right) ; b_{2,1}=I_{1}\left(\omega_{1}\right) ; b_{2,2}=K_{1}\left(\omega_{1}\right) ; b_{2,3}=-K_{1}\left(\omega_{2}\right) ; \\
b_{3,1} & =\kappa_{1}^{2} \omega_{1} I_{0}\left(\omega_{1}\right)-2 I_{1}\left(\omega_{1}\right) ; b_{3,2}=-\kappa_{1}^{2} \omega_{1} K_{0}\left(\omega_{1}\right)-2 K_{1}\left(\omega_{1}\right) ; b_{3,3}=\tilde{\mu}_{2}\left(\kappa_{2}^{2} \omega_{2} K_{0}\left(\omega_{2}\right)+2 K_{1}\left(\omega_{2}\right)\right), \tilde{\mu}_{2}=\mu_{2} / \mu_{1} ; \\
H_{1, n} & =-\frac{p_{n}}{\mu_{1}}-\sum_{j=1}^{n} C_{n-j}^{(1)}\left[\kappa_{1}^{2} G_{j}^{\prime}\left(\omega_{1} \rho_{0}\right)+\left(\kappa_{1}^{2}-2\right) G_{j}\left(\omega_{1} \rho_{0}\right)\right]-\sum_{j=1}^{n} D_{n-j}^{(1)}\left[\kappa_{1}^{2} W_{j}^{\prime}\left(\omega_{1} \rho_{0}\right)+\left(\kappa_{1}^{2}-2\right) W_{j}\left(\omega_{1} \rho_{0}\right)\right] ; \\
H_{2, n} & =\sum_{j=1}^{n}\left[D_{n-j}^{(2)} W_{j}\left(\omega_{2}\right)-C_{n-j}^{(1)} G_{j}\left(\omega_{1}\right)-D_{n-j}^{(1)} W_{j}\left(\omega_{1}\right)\right] ; H_{3, n}=\tilde{\mu}_{2} \sum_{j=1}^{n} D_{n-j}^{(2)}\left[\kappa_{2}^{2} W_{j}^{\prime}\left(\omega_{2}\right)+\left(\kappa_{2}^{2}-2\right) W_{j}\left(\omega_{2}\right)\right]- \\
& -\sum_{j=1}^{n} C_{n-j}^{(1)}\left[\kappa_{1}^{2} G_{j}^{\prime}\left(\omega_{1}\right)+\left(\kappa_{1}^{2}-2\right) G_{j}\left(\omega_{1}\right)\right]-\sum_{j=1}^{n} D_{n-j}^{(1)}\left[\kappa_{1}^{2} W_{j}^{\prime}\left(\omega_{1}\right)+\left(\kappa_{1}^{2}-2\right) W_{j}\left(\omega_{1}\right)\right],
\end{aligned}
$$


$G_{j}^{\prime}\left(\omega_{i} \rho\right)=\sum_{p=0}^{j} a_{j, p} \frac{\left(\omega_{i} \rho\right)^{p}}{2^{p} p !}\left[\omega_{i} I_{p}\left(\omega_{i} \rho\right)-\frac{I_{p+1}\left(\omega_{i} \rho\right)}{\rho}\right], W_{j}^{\prime}\left(\omega_{i} \rho\right)=\sum_{p=0}^{j} a_{j, p} \frac{\left(-\omega_{i} \rho\right)^{p}}{2^{p} p !}\left[-\omega_{i} K_{p}\left(\omega_{i} \rho\right)-\frac{K_{p+1}\left(\omega_{i} \rho\right)}{\rho}\right]$

From (15) obtain the recurrent solution:

$D_{n}^{(2)}=\frac{\left(H_{n, 1} b_{2,1}-H_{n, 2} b_{1,1}\right)\left(b_{1,2} b_{3,1}-b_{3,2} b_{1,1}\right)-\left(H_{n, 1} b_{3,1}-H_{n, 3} b_{1,1}\right)\left(b_{1,2} b_{2,1}-b_{2,2} b_{1,1}\right)}{b_{1,1}\left(b_{2,3}\left(b_{3,2} b_{1,1}-b_{1,2} b_{3,1}\right)+b_{3,3}\left(b_{1,2} b_{2,1}-b_{2,2} b_{1,1}\right)\right\}} ; D_{n}^{(1)}=\frac{H_{n, 1} b_{2,1}-H_{n, 2} b_{1,1}+b_{2,3} b_{2,1} D_{n}^{(2)}}{b_{1,2} b_{2,1}-b_{2,2} b_{1,1}} ;$

$C_{n}^{(1)}=\frac{H_{n, 1}-b_{1,2} D_{n}^{(1)}}{b_{1,1}}, \quad n=0,1,2, \ldots$

Having gradually defined with the help of recurrent solutions (16) all $C_{n-j}^{(i)}, D_{n-j}^{(i)}$, we will get the final problem solution as:

$$
\begin{aligned}
& u^{(1)}(\rho, \tau)=\lambda \sum_{n=0}^{\infty} L_{n}(\lambda \tau) \sum_{j=0}^{n}\left[C_{n-j}^{(1)} G_{j}(\lambda \rho)+D_{n-j}^{(1)} W_{j}(\lambda \rho)\right] \\
& u^{(2)}(\rho, \tau)=\lambda \sum_{n=0}^{\infty} L_{n}(\lambda \tau) \sum_{j=0}^{n} D_{n-j}^{(2)} W_{j}\left(\lambda \tilde{c}_{2} \rho\right)
\end{aligned}
$$

Parameter $\lambda$ serves as the scale multiplier in numerical summation of the series (17).

\section{NUMERICAL ANALYSIS}

For the purpose of approbation of the received results, a comparative analysis of numerical results obtained from the correlations (17) with known results for a homogeneous cylinder (6) received using the integral Laplace transform, was conducted.

A solution for a homogeneous cylinder can be obtained from the correlations (17) if to consider that the cylinder is in contact with space, constants and density of which are significantly lower than corresponding values of the cylinder material.

For the numerical analysis it was selected a cylinder with a relative radius of the inner surface $\rho_{0}=0.6$ and $\kappa_{1}^{2}=3.5$ which is affected by external load:

$p^{*}(\tau)=p^{*} \times\left(1-\exp \left(-\tau_{0} \tau\right)\right)^{2}$,

where $\mathrm{p}^{*}$ - dimensional value $(\mathrm{Pa})$.

Dependance (18) makes it possible to agree well zero initial conditions with boundary ones, and in this case parameter $\tau_{0}$ determines the time of the external load output on the stationary value.

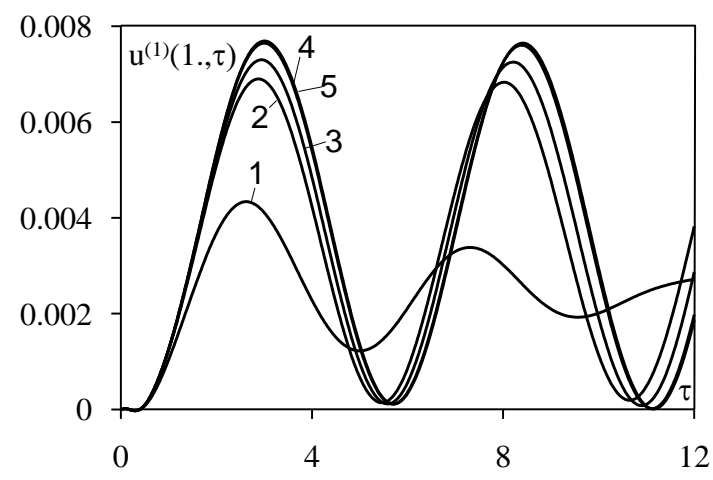

Fig. 2. Displacements the outer surface of the cylinder with different mechanical properties of elastic space

In the Fig. 2 there is the time distribution of dimensionless displacmenents $u^{(1)}(\rho, \tau)$ on the surface $\rho=1$ under $\kappa_{2}^{2}=2.5$ and different relative mechanical properties of space: $\tilde{\mu}_{2}=\tilde{c}_{2}=$ $0.5,0.1,0.05,0.01,0.005$, correspondingly curves $1,2,3$, 4,5 . Calculations were performed as $\tau_{0}=3$ and in the series according to the Laguerre polynomials 60 members were held.

As it is seen, the reduction of relative mechanical properties of the space leads to the increase in the amplitude of oscillation and the termination of the process of wave attenuation that agrees well with the physics of the phenomenon.

The results of calculation obtained for the value when were compared in their turn with the results obtained for a homogeneous cylinder using the Laplace transform (Sneddon, 1951). It was found out, that holding 60 members of the series according to the Laguerre polynomials the relative error between the results received using two methods does not exceed $0.5 \%$.

Using the results obtained for the case of the cylinder and space it was also performed the calculation of the stress-strain state in the thin-walled steel cylinder $\left(\rho_{0}=0.9, \kappa_{1}^{2}=3.5\right)$, inserted into the space of the sandstone $\left(\kappa_{2}^{2}=2.7, \tilde{\mathrm{c}}_{2}=0.67\right.$, $\left.\tilde{\mu}_{2}=0.16\right)$.

In this case it was considered that the load of the cylinder inner surface is a function of the impulsing tupe:

$p(\tau)=p^{*}\left((1-\tau)^{2}-1\right)^{2}, \tau \leq 2 ; \quad p(\tau)=0, \tau>2$.

In the Fig. 3 there is a time distribution of dimensionless radial stresses $\sigma_{\rho}=\sigma_{\rho \rho}^{(i)}(\rho, \tau) / p^{*}$ at different points of the cylinder and space. In this case, given the results of the comparative analysis above 60 members of the series according to the Laguerre polynomials were held.

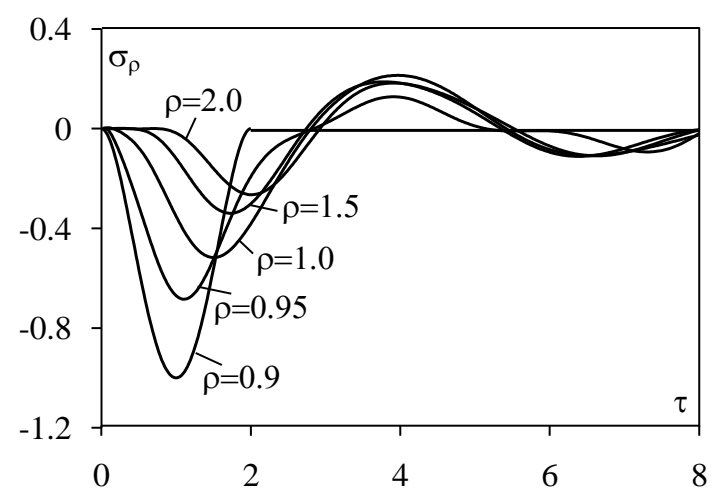

Fig. 3. Time distribution of radial stresses on different surfaces

According to the given results, the specified stresses reach the maximum modulo value on the surface where there is a load. On the division surface of cylinder materials and external space ( $\rho=1)$ during the load impulse action radial stresses make about $50 \%$ of its level and after the time moment $\tau=3$ thay change their sign and quickly attenuate. Approximately the same conclusions can be reached about the radial stresses in the material of the space. 
Fig. 4 presents the displacements change at different point of the cylinder for $p^{*} / \mu_{1}=0.01$.

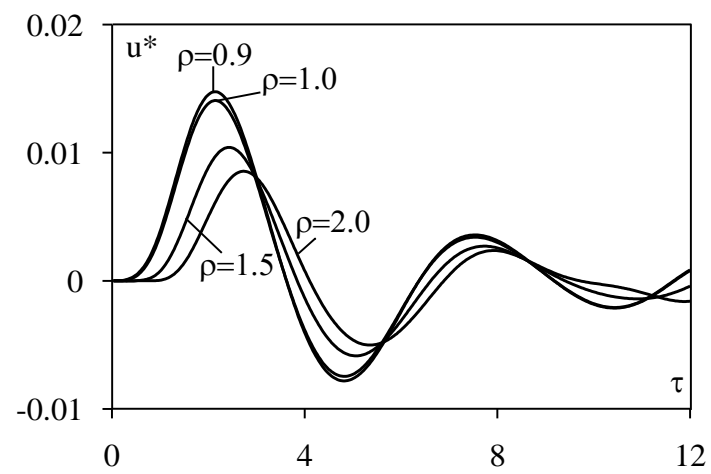

Fig. 4. Time distribution of radial displacements

As it is seen from the above, displacements of two boundary surfaces almost coincide that agrees well with small relative thickness of the cylinder and the malleability of the space.

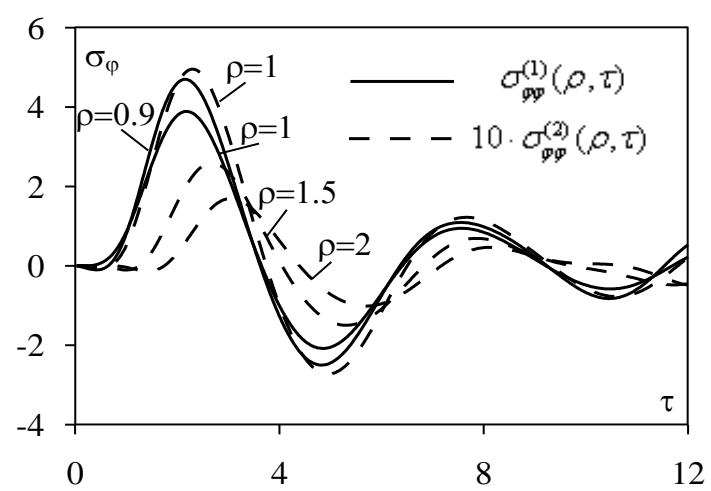

Fig. 5. Time distribution of radial stresses

The results of the calculation of dimensionless circular stresses $\sigma_{\phi} \equiv \sigma_{\phi \phi}^{(i)} / p^{*}$ are given presented in Fig. 5. At that stresses acting in the space were magnified 10 times.

According to the given results, circular stresses in the cylinder in absolute value exceed correspondent radial ones almost 5 times. In the space the level of these stresses slumps due to significantly poorer elastic properties of its material. Circular stresses reach the maximum value at the moments of time that follow immediately after the load impulse action termination and qualitatively repeat time distribution of radial displacements.

\section{CONCLUSION}

The paper proposes a new solution of the plane dynamic problem of elasticity theory for a elastic space with cylindrical tab. The solution is obtained as series of Laguerre polynomials, which coefficients are found from recurrent relations. The results of numerical analysis of transient stress-strain state in elastic space with cylindrical insertion might be used for the technological process of hydraulic fracturing during shale gas extraction.

\section{REFERENCES}

1. Dai H., Wang X. (2005), Thermo-electro-elastic transient responses in piezoelectric hollow structures, International Journal of Solids and Structures, 42, 1151-1171.

2. Filippo de Monte (2006), Multi-layer transient heat conduction using transition time scales, International Journal of Thermal Science, 45, 882-892.

3. Liu G., Qu J. (1998), Transient Wave Propagation in a Circular Annulus Subjected to Transient Excitation on Its Outer Surface, Journal of the Acoustical Society of America, 104, 1210-1220.

4. Lu X., Tervola P., Viljanen M. (2006), Transient analytical solution to heat conduction in composite circular cylinder, International Journal of Heat and Mass Transfer, 49, 341-348.

5. Onyshko L.I., Senyuk M.M. (2009), Stressed state of a hollow twolayer cylinder under dynamic loads, Material Science, 45, 55-61.

6. Savruk M., Onyshko L., Senyuk M. (2008), A plane dynamic axisymmetric problem for a hollow cylinder, Materials Science, 1-9.

7. Sladek V., Sladek J., Zhang C. (2008), Computation of stresses in non-homogeneous elastic solids by local integral equation method: a comparative study, Computational Mechanics, 41, 827-845.

8. Sneddon I. (1951), Fourier transforms, McCraw-Hill Book Company, New York.

9. Sulym H., Hutsaylyuk V., Pasternak la., Turchyn I. (2013), Stressstrain state of an elastic rectangular plate under dynamic load, Mechanika, 19, 620-626.

10. Sulym H., Turchyn I. (2014), Axisymmetric quasistatic thermal stressed state in a half space with coating, Journal of Mathematical Science, 198, 103-117.

11. Theotokoglou E., Stampouloglou I. (2008), The radially nonhomogeneous elastic axisymmentric problem, International Journal of Solids and Structures, 45, 6535-6552.

12. Turchyn I., Turchyn $\mathbf{0}$. (2013), Transient plane waves in multilayered half-space, Acta Mechanica et Automatica, 7, 53-57.

13. Wang X., Lu G., Guillow S. (2002), Stress wave propagation in orthotropic laminated thick-walled spherical shells, International Journal of Solids and Structures, 39, 4027-4037.

14. Yin X.C., Yue Z.Q. (2002), Transient plane-strain response of multilayered elastic cylinders to axisymmetric impulse, Journal of Applied Mechanics, 69, 825-835.

15. Zhang X., Hasebe N. (1999), Elasticity solution for a radially nonhomogeneous hollow cylinder, Journal of Applied Mechanics, 66, 598-606. 